\title{
Decomposing Public Opinion Variation into Ideology, Idiosyncrasy, and Instability
}

\author{
Benjamin E. Lauderdale, London School of Economics and Political Science \\ Chris Hanretty, Royal Holloway, University of London \\ Nick Vivyan, Durham University
}

We propose a method for decomposing variation in the issue preferences that US citizens express on surveys into three sources of variability that correspond to major threads in public opinion research. We find that, averaging across a set of high-profile US political issues, a single ideological dimension accounts for about 1/7 of opinion variation, individuals' idiosyncratic preferences account for about 3/7, and response instability for the remaining $3 / 7$. These shares vary substantially across issue types, and the average share attributable to ideology doubles when a second ideological dimension is permitted. We also find that (unidimensional) ideology accounts for almost twice as much response variation (and response instability is substantially lower) among respondents with high, rather than low, political knowledge. Our estimation strategy is based on an ordinal probit model with random effects and is applicable to other data sets that include repeated measurements of ordinal issue position data.

S ince Converse (1964) political scientists have debated the extent to which citizens have organized preferences about political issues or indeed whether they have such preferences at all (Achen 1975; Zaller 1992; Zaller and Feldman 1992). In this short article, we exploit recent survey data collected by Broockman (2016) to decompose variation in citizens' expressed issue preferences into three sources of variability. First, how much is ideological, by which we mean predictable from a citizen's preferences on other issues given the way preferences are typically organized in the population? ${ }^{1}$ Second, how much is idiosyncratic, by which we mean preferences that are not predictable from a citizen's preferences on other issues but that are nonetheless stable in repeated measurement? Third, how much is instability, by which we mean preference variation that is not stable in repeated measurement and that combines measurement error and nonattitudinal response behaviors? Although scholars have at various points argued for the relative importance of each of these sources of variability in opinion, to our knowledge none have provided a quantitative summary of how much variation is attributable to each one.

\section{CONTEXT}

There is a long-running debate within political science about the extent to which most citizens have "reasonably wellformed attitudes on major political issues" (Zaller and Feldman 1992, 579) that exist independently of the survey instruments designed to measure such attitudes. On one view, survey respondents may be perfectly capable of responding to questions designed to elicit political preferences, but these responses merely reflect the result of a sample of considerations bearing on the matter, which are context biased in ways that lead them to give different answers at different points in time (579). Evidence in favor of this view comes from the low

Benjamin E. Lauderdale (b.e.lauderdale@lse.ac.uk) is an associate professor in the Department of Methodology at the London School of Economics and Political Science, London, UK. Chris Hanretty (Chris.Hanretty@rhul.ac.uk) is a professor in the Department of Politics and International Relations at Royal Holloway, University of London, Egham, Surrey, UK. Nick Vivyan (nick.vivyan@durham.ac.uk) is an associate professor in the School of Government and International Affairs at Durham University, Durham, UK.

Data and supporting materials necessary to reproduce the numerical results in the article are available in the JOP Dataverse (https://dataverse.harvard.edu /dataverse/jop). An online appendix with supplementary material is available at http://dx.doi.org/10.1086/695673.

1. This is a thin definition of ideology - what Converse refers to as constraint. 
correlation between many measures of attitudes at different points in time, from open-ended survey questions eliciting voter considerations, and from the extensive literature on priming. Against this evidence, it has been argued that low over-time correlation is an indication that survey responses are subject to large measurement error such that stable underlying attitudes can result in volatile survey responses (Achen 1975) and that many priming effects are better understood as information effects (Lenz 2009).

If citizens do not have stable preferences on major political issues at all, then there is no point arguing about whether those attitudes can be effectively summarized by a position in some latent ideological space. However, if political preferences are at least partially stable or real, then they may or may not have a low dimensional structure (ideology) that explains much of the variation in preferences across different issues. There is disagreement about whether preferences can be represented using just one left-right dimension (Jacoby 1995; Jost, Federico, and Napier 2009) or whether they must instead be represented using two dimensions (Duckitt and Sibley 2009; Evans, Heath, and Lalljee 1996; Feldman and Johnston 2014). The more that preferences can be represented in terms of a small number of underlying dimensions, the less role there is for idiosyncratic preferences, understood as "nonideological" preferences that cannot be predicted on the basis of preferences about other issues but that are nonetheless real, stable views about particular issues (Broockman 2016).

This article argues that we can usefully understand these two debates - about the extent to which preferences are real and about the extent to which they are ideological — as part of a larger question about the composition of survey response variation. Our decomposition of preferences into components corresponding to ideology, idiosyncrasy, and instability can be mapped onto these previous debates. The debate over whether preferences are real is a debate about the relative contribution of ideology plus idiosyncrasy versus instability. The debate over whether preferences are ideological is a debate about the relative contribution of ideology versus idiosyncrasy (either with or without instability).

In our reading, no one has directly assessed the three-way decomposition quantitatively. Converse (1964) evaluates both the correlation structure across issues as well as the stability of responses within issues, but the synthesis of the evidence from those analyses is qualitative. Subsequent studies, including the Broockman study whose data we reanalyze, have similarly done a series of analyses and then summarized the results qualitatively. The costs of a purely qualitative synthesis of the evidence are apparent in the subsequent multidecade debate spurred by Converse's study, a debate that tended to get simplified into binary questions about whether preferences were real or not, or ideological or not, rather than decompositional questions about how much of observed preference variation is real and how much is ideological (Converse 2000; Feldman 2013).

\section{DATA}

To quantitatively decompose response variation into ideology, idiosyncrasy, and instability, we need data with several features. Repeated measurements on the same respondents must be taken in order to distinguish stable preferences from response instability. Questions on a range of political issues must be asked in order to assess whether preferences are ideologically structured. Anchored response scales are desirable in order to limit measurement error associated with differential scale use, both across respondents and within respondents across repeated measurements.

Fortunately, data satisfying all of these criteria are provided by Broockman (2016). Broockman surveyed US citizens in early January and again in late February 2014. He asked eight questions as part of a political knowledge battery and 13 ordinal questions on particular policies. The questions covered both economic issues (health care, taxes, Medicare, unions, education, social security) and social/ cultural issues (gun control, immigration, abortion, the environment, gay rights, affirmative action, and contraception). The responses to the ordinal policy questions were constructed so that the middle option represented the status quo, and the next least extreme response options captured the median position of the Democratic and Republican parties respectively. Remaining options represented more extreme positions. ${ }^{2}$ Giving such specific response options mitigates the risk that variation in manifest survey responses will result from the way respondents use response scales rather than from respondents' varying underlying preferences.

Although we focus on the policy questions, we also use Broockman's latent trait measure of political knowledge. Correct or incorrect answers to eight knowledge questions were aggregated, allowing for variation in question difficulty and the ability of each question to discriminate between respondents with otherwise similar levels of knowledge. For simplicity, we categorize respondents as "high" or "low" political knowledge, using the median political knowledge score as the cutoff. A total of 1,137 respondents participated in the first wave, of whom 513 also participated in the second wave. Average levels of political knowledge were modestly

2. Response options were ordered from left- to right-wing for some questions and right- to left-wing for others. 
(0.15 SD) higher among wave 2 respondents than among respondents who only responded to wave 1 .

\section{METHOD}

Decomposing variation in ordinal response data requires us to make some scaling and modeling assumptions. We assume an ordinal probit response model, where respondents have latent continuous responses $\left(Y^{\star}\right)$ that map on to manifest categorical responses $(Y)$ according to cutpoints, which are drawn from a uniform prior subject to the ordering constraint. Our model for the latent response takes the form:

$$
Y_{i j t}^{\star}=\beta_{j} \theta_{i}+\nu_{i j}+\varepsilon_{i j t},
$$

where $i$ indexes respondents, $j$ indexes questions, $t$ indexes the survey wave $(t=1,2)$, and the parameters in the model are drawn $\theta_{i} \sim N(0,1), \nu_{i j} \sim N\left(0, \omega_{j}^{2}\right)$, and $\varepsilon_{i j t} \sim N\left(0, \sigma_{j}^{2}\right)$.

The first term in the model $\left(\beta_{j} \theta_{i}\right)$ is the ideological component, operationalized as a unidimensional spatial model where $\beta_{j}$ describes how the respondent's latent ideal point $\theta$ predicts her latent response on issue $j$. The signs of $\theta$ and $\beta$ are both arbitrary and unimportant for the variance decomposition. The second term in the model $\left(\nu_{i j}\right)$ is a respondentby-issue random effect. This term captures idiosyncratic variation in preferences that is not attributable to ideology but that is nonetheless stable across repeat measurements in different time periods $t$. The third term in the model $\left(\varepsilon_{i j t}\right)$ is an error term capturing instability, variation in an individuals' manifest responses across repeated measurements. This instability could include response changes from several hypothesized mechanisms, including instability as sheer error (Achen 1975), as sampling from top-of-the-head considerations (Zaller and Feldman 1992), as "flipping a coin" (Converse 1964), or as "real" opinion change in the brief time between survey waves (which we discuss further in the conclusion).

We are not interested in the sign or magnitude of these terms for any individual respondent but rather in their variance per survey item across respondents. In the case of the respondent-by-issue random effect and the error term, we use $\omega_{j}^{2}$ and $\sigma_{j}^{2}$ to refer to these variances. The variance of the ideological component is $\beta_{j}^{2}$, which follows from the identity $\operatorname{Var}(c x)=c^{2} \operatorname{Var}(x)$ and the fact that $\theta_{i} \sim N(0,1)$.

Because these sources of variation are independent by assumption, the total variance per survey item can be expressed as $\sum_{j}=\beta_{j}^{2}+\omega_{j}^{2}+\sigma_{j}^{2}$. The share of the total variance attributable to ideology can therefore be expressed as $\beta_{j}^{2} / \sum_{j}$; the share attributable to idiosyncrasy, as $\omega_{j}^{2} / \sum_{j}$; and that attributable to instability, $\sigma_{j}^{2} / \sum_{j}$. This three-way decomposition of latent scale variance is similar in spirit to the McKelvey and Zavoina (1975) pseudo- $R^{2}$ statistic for the ordinal probit regression model, which estimates the $R^{2}$ that would be re- covered via linear regression on the unobserved latent variable.

The priors for $\beta_{j}, \omega_{j}$, and $\sigma_{j}$ are standard normal, half normal, and half normal, respectively. These priors are chosen so that the prior over the decomposition $\left(\beta_{j}^{2} / \sum_{j}, \omega_{j}^{2} / \sum_{j}, \sigma_{j}^{2} / \sum_{j}\right)$ for each issue $j$ is a symmetric Dirichlet distribution with parameters equal to $1 / 2$, which is Jeffrey's noninformative prior. ${ }^{3}$ These priors correspond to a weak prior expectation for an equal split of the variance among ideology, idiosyncrasy, and instability, and any deviation from this in our results reflects evidence from the data. The model was estimated using Stan 2.16.2 (Carpenter et al. 2017).

\section{RESULTS}

Figure 1 shows the estimated composition of opinion variation for each issue in the data set that we examine. Averaging across issues, we estimate that the fraction of variation accounted for by ideology is low (14\%; $95 \%$ confidence interval, $11 \%-17 \%)$ when compared to that explained by idiosyncrasy $(42 \%$; $95 \%$ confidence interval, $38 \%-45 \%)$ or instability (45\%; 95\% confidence interval, $41 \%-48 \%)$. Most of the stable preference variation across individuals and issues is not organized along a single political dimension.

The four most ideological issues are those related to the size of government: Medicare, taxes, social security, and health. However, the four issues with the most stable preferences are mostly different issues: immigration, marijuana, gay rights, and health. This highlights the distinction between citizens having stable preferences on issues and having ideological preferences. Respondents to this study have real and measurable views about marijuana and immigration (relatively low instability), but those views are almost entirely unpredictable on the basis of a unidimensional summary of their other positions. Stated preferences regarding unions (and to a lesser extent education and the environment) are both lacking in ideological structure and highly unstable; these are the issues on which the most respondents seem to lack real preferences.

We have conducted a large number of robustness checks on variant forms of the model and data set (a full tabulation of these is provided in an appendix, available online). When we simulate data from the estimates and data-generating process of the model and refit the model, we recover the estimated parameter values and decomposition almost exactly, so there is negligible estimator bias. If we omit the spatial term and decompose preference variation into only

3. If $\beta_{j}, \omega_{j}$, and $\sigma_{j}$ have prior distributions that are standard (half) normal, their squares are distributed $\chi_{1}^{2}$, which is equivalent to $\Gamma(1 / 2,2)$. The simplex constructed by dividing three $\Gamma(1 / 2,2)$ random variables by their sum is distributed $D(1 / 2,1 / 2,1 / 2)$. 


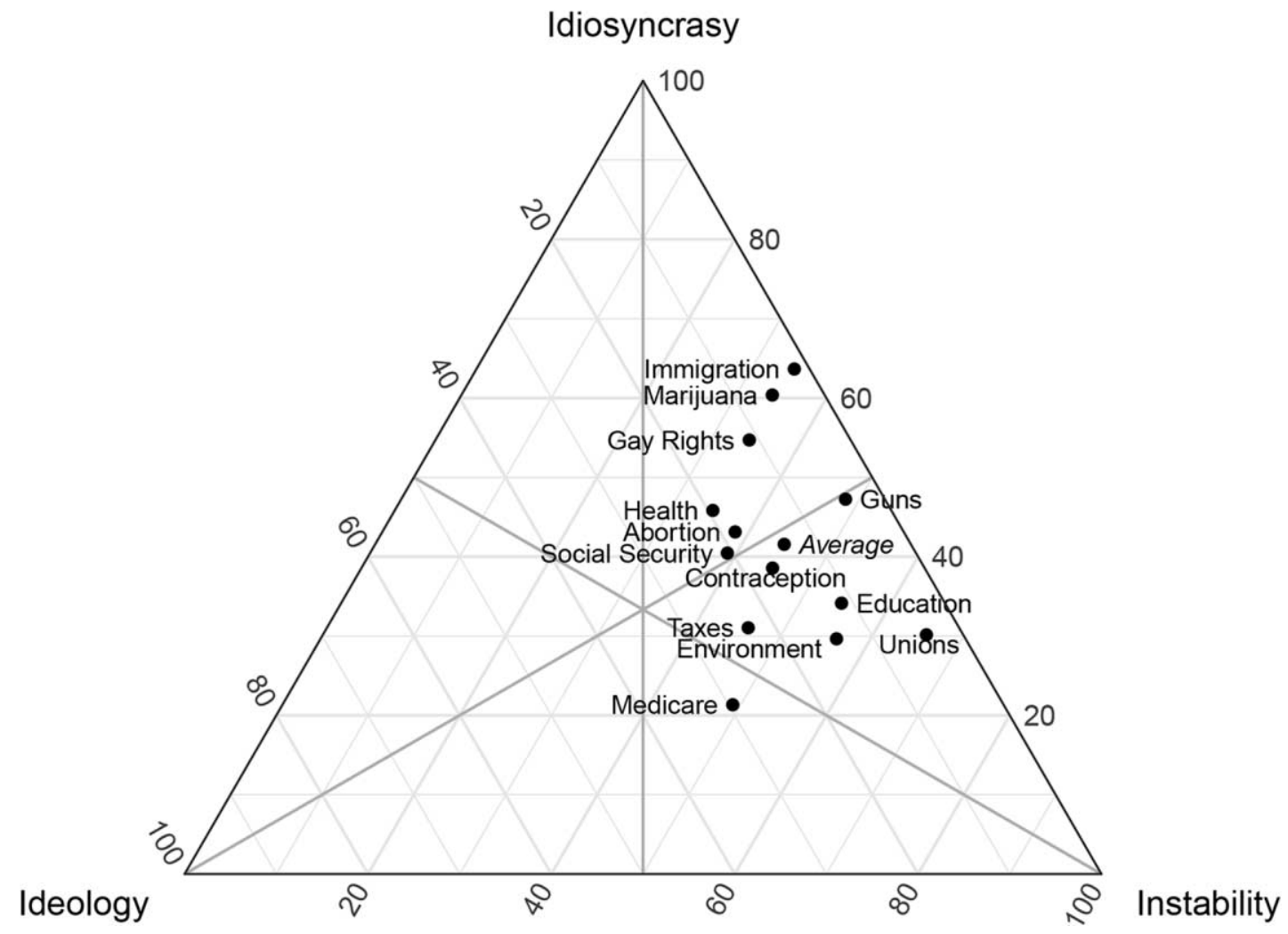

Figure 1. Ternary plot showing the composition of the variance for each issue. Medicare is the most ideological issue, immigration is the most idiosyncratic, unions is the most unstable.

idiosyncrasy and instability, we get nearly identical estimates for instability, and the estimates of idiosyncrasy absorb the variation associated with ideology in the full model. If we omit the idiosyncrasy term instead, the share estimated for ideology absorbs some of the stable variation, increasing from $14 \%$ to $18 \%$.

If we analyze only the first wave of data, we get nearly the same estimate for the share of variation associated with ideology, but we can no longer distinguish between idiosyncrasy and instability. For comparison purposes, this onewave model can also be applied to Broockman's accompanying survey of state legislators. Averaging across the policy issues about which both state legislators and citizens were asked, we estimate that ideology accounts for $55 \%$ of the variation among state legislators, versus $15 \%$ for citizens. This contrast illustrates that it is possible to estimate high shares of variation for ideology under this approach, as well as to quantify the well-known, very large difference between the degree of spatial structure in the positions of elites and citizens.

Adding a second spatial dimension to the ideological component of the model increases the fraction of variation explained by ideology from $14 \%$ to $24 \%$ (95\%; confidence interval, $21 \%-28 \%$ ), while leaving that due to instability un- changed. The fact that adding a second dimension nearly doubles the variance explained by ideology reinforces the point that a modest fraction of the variation in public opinion is captured by a single dimension, despite a two-party system that one might expect to organize opinion unidimensionally.

Our estimates are somewhat sensitive to collapsing the extreme policy categories, with the ideology component rising to $16 \%$ when we collapse the two most extreme categories on each side to generate five-point scales and $20 \%$ when we collapse all categories on each side of the central status quo category to generate three-point scales. The increase in the ideology component is entirely at the expense of the inconsistency component, indicating that there is more structure and stability in the direction of change that respondents would like than in exactly how far they would like policy to move. Finally, we have analyzed a version of the model where $\theta_{i}$ is allowed to change at the individual level between the two periods as an alternative to the unstructured change captured by the instability term, but we see very little evidence of ideologically coherent movement between these two survey waves.

Previous research has suggested that individuals fall into different groups that exhibit different levels of opinion sta- 
bility (Hill 2001; Hill and Kriesi 2001) and opinion structure. Figure 2 shows that, when we split the sample into low- and high-knowledge individuals, and allow for different ideological dimensions in each half of the sample, we find that high-knowledge individuals have more ideologically structured preferences on all but two issues and have less response instability on all issues. Averaging across issues, ideological structure explains $23 \%$ of variation among high-knowledge respondents versus $12 \%$ among low-knowledge respondents, with a $95 \%$ posterior interval for the difference running from $6 \%$ to $16 \%$. Response instability accounts for $38 \%$ of response variation among high-knowledge respondents versus 51\% among low-knowledge respondents, with a 95\% posterior interval for the difference running from $-19 \%$ to $-8 \%$. The single ideological dimensions that best predict responses among low- and high-knowledge respondents are different: among low-knowledge respondents, attitudes on guns and unions most strongly predict other issue preferences, whereas they are among the least predictive among high-knowledge respondents.

\section{DISCUSSION AND CONCLUSION}

These estimates will not come as a great shock to public opinion researchers. As we noted at the outset, the ideas that citizens do not organize their views as strongly as political parties and elites and are sometimes unstable in the preferences they express are hardly novel. The value of this article is in providing a quantitative decomposition: how much of the preference variation we see is due to each of these factors? On average, in a particular survey instrument que- rying a particular set of issues at a particular moment in US history, about $1 / 7$ of cross-sectional variation reflects a common ideological dimension of variation, $3 / 7$ is real but idiosyncratic variation in individuals' views, and $3 / 7$ is response instability.

The substantial variation across political issues in the degree to which citizens have "real preferences" (either ideological or idiosyncratic), plus the further variation in the extent to which those preferences are ideological rather than idiosyncratic, indicates that our results might change substantially with a different sample of issues. Our results might also change with different response categories or different wordings of those response categories - we note Broockman's own cautionary remarks regarding the choice of policy options $(2016,187)$ - but we have no reason to believe that differently worded response options would affect our results in any particular direction. The issues chosen include a variety of types (some primarily economic, some primarily sociocultural), and all of the issues are sufficiently prominent for researchers to be able to identify the preferred positions of elite actors (Broockman 2016). If lower profile issues were chosen instead, we would expect the estimated ideological and idiosyncratic shares of the decomposition to decline and instability to increase.

The variation we observe by political knowledge level suggests that the results might also vary in a more representative sample. Those who responded to the second survey wave had somewhat higher levels of knowledge than those who only responded to the first wave. This suggests that our findings, if anything, overestimate the portion of variation

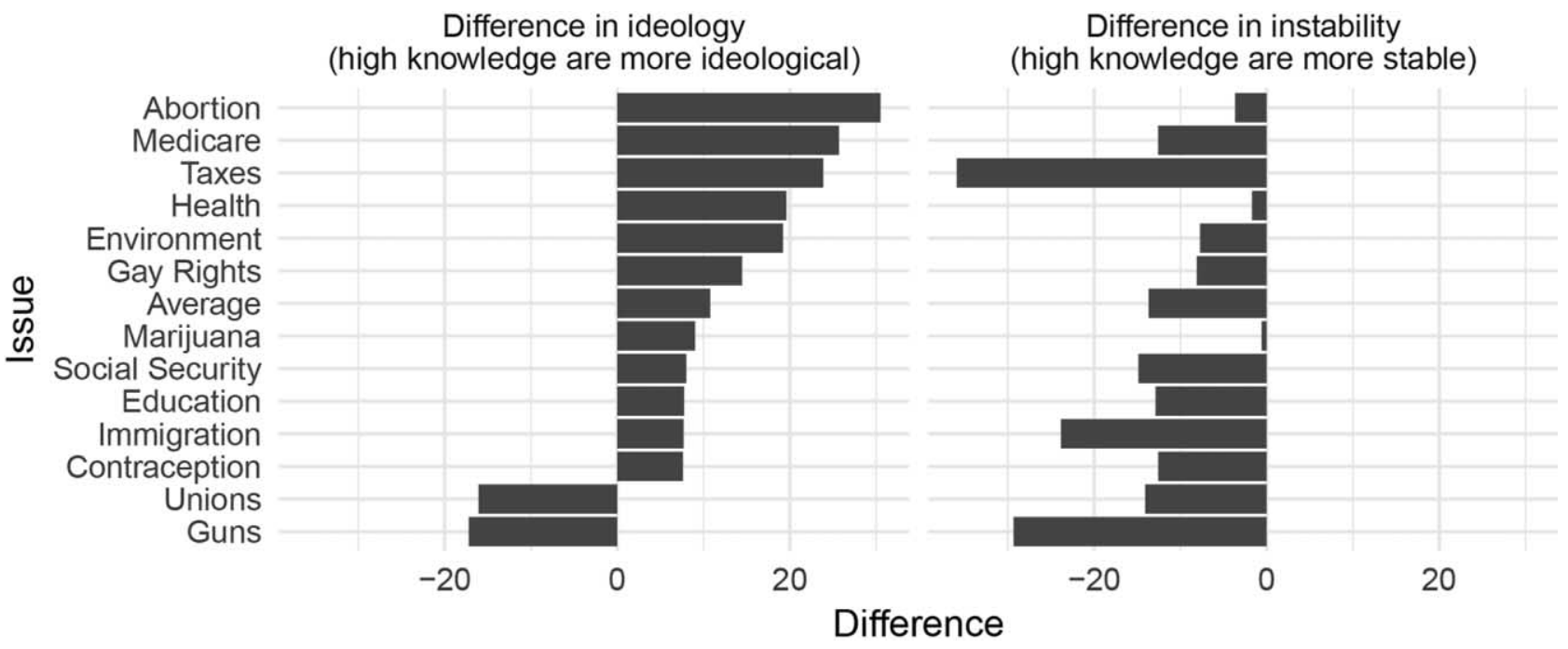

Figure 2. Difference in the percentage of opinion variation explained by ideology and idiosyncrasy for high-knowledge respondents versus low-knowledge respondents. 
accounted for by ideology and underestimate the portion of variation accounted for by instability.

In addition to the ways that our estimates are context dependent because of the structure of the survey data we work with, they are also model dependent because of the way we structure the analysis. While the model we specify enables an elegant variance decomposition, it makes certain functional form assumptions in order to enable that decomposition. While we think these are fairly innocuous with respect to the general conclusions, the exact estimates would surely change under a different approach to decomposition. Because the data we work with have only two waves, we also cannot distinguish instability from real opinion change, which is a fourth potential source of variation in response, albeit one that is only likely to be more important over timescales longer than six weeks. The framework described above can be easily extended to perform a four-way decomposition given additional survey waves. The key to doing so is the logic used by Converse (1964): if opinion change is real, opinions in adjacent waves will be more similar than those in nonadjacent waves. In the context of our model, this would most naturally take the form of a normally distributed random-walk process, for each respondent, for each item. We have also set aside possible opinion processes that would fundamentally undermine the logic of this sort of decomposition, such as the possibility that opinion on one issue might predict changes in opinion on other issues across waves (dynamic constraint).

In sum, our findings put quantitative figures behind the argument made by Broockman (2016) that real idiosyncratic preference variation is much greater than real ideological preference variation among the US public. This is important not only for adjudicating between theories of public opinion but also because of the widespread use of unidimensional models, both formal and informal, to understand interactions between elites and the public. The fact that roughly $3 / 4$ of real public opinion variation does not reflect a unidimensional spatial dimension, and the fact that a second spatial dimension is nearly as powerful as the first, does not necessarily undermine the use of such models. There is, by construction, always a single dimension that captures more opinion variation than any other, and positions on that dimension will therefore always be important to electoral interactions. However, conclusions drawn from models focusing exclusively on a single dimension may or may not be robust to the fact that this dimension captures only a small proportion of the real opinion variation on which basis citizens may make choices and to which elites may have opportunities to respond.

\section{ACKNOWLEDGMENTS}

We thank seminar participants at University College London and Humboldt University for helpful comments and suggestions.

\section{REFERENCES}

Achen, Christopher H. 1975. "Mass Political Attitudes and the Survey Response.” American Political Science Review 69 (4): 1218-31.

Broockman, David E. 2016. "Approaches to Studying Policy Representation.” Legislative Studies Quarterly 41 (1): 181-215.

Carpenter, Bob, Andrew Gelman, Matt Hoffman, Daniel Lee, Ben Goodrich, Michael Betancourt, Michael A. Brubaker, Jiqiang Guo, Peter Li, and Allen Riddell. 2017. "Stan: A Probabilistic Programming Language.” Journal of Statistical Software 76 (1). doi:10.18637/ jss.v076.i01.

Converse, Philip E. 1964. "The Nature of Belief Systems in Mass Publics." In David Apter, ed., Ideology and Its Discontents. New York: Glencoe Free Press.

Converse, Philip E. 2000. "Assessing the Capacity of Mass Electorates." Annual Review of Political Science 3:331-53.

Duckitt, John, and Chris G. Sibley. 2009. "A Dual-Process Motivational Model of Ideology, Politics, and Prejudice." Psychological Inquiry 20 (2-3): 98-109.

Evans, Geoffrey, Anthony Heath, and Mansur Lalljee. 1996. "Measuring Left-Right and Libertarian-Authoritarian Values in the British Electorate." British Journal of Sociology 47 (1): 93-112.

Feldman, Stanley. 2013. "Political Ideology." In L. Huddy, D. O. Sears, and J. S. Levy, eds., The Oxford Handbook of Political Psychology. Oxford: Oxford University Press.

Feldman, Stanley, and Christopher Johnston. 2014. "Understanding the Determinants of Political Ideology: Implications of Structural Complexity." Political Psychology 35 (3): 337-58.

Hill, Jennifer L. 2001. "An Extension and Test of Converse's Black-andWhite Model of Response Stability." American Political Science Review 95 (2): 397-413.

Hill, Jennifer L., and Hanspeter Kriesi. 2001. "Classification by OpinionChanging Behavior: A Mixture Model Approach.” Political Analysis 9 (4): 301-24.

Jacoby, William G. 1995. "The Structure of Ideological Thinking in the American Electorate." American Journal of Political Science 39 (2): 314-35.

Jost, John T., Christopher M. Federico, and Jaime L. Napier. 2009. "Political Ideology: Its Structure, Functions, and Elective Affinities." Annual Review of Psychology 60:307-37.

Lenz, Gabriel S. 2009. "Learning and Opinion Change, Not Priming: Reconsidering the Priming Hypothesis." American Journal of Political Science 53 (4): 821-37.

McKelvey, Richard D., and William Zavoina. 1975. "A Statistical Model for the Analysis of Ordinal Level Dependent Variables.” Journal of Mathematical Sociology 4 (1): 103-20.

Zaller, John. 1992. The Nature and Origins of Mass Opinion. Cambridge: Cambridge University Press.

Zaller, John, and Stanley Feldman. 1992. "A Simple Theory of the Survey Response: Answering Questions versus Revealing Preferences.” American Journal of Political Science 36 (3): 579-616. 